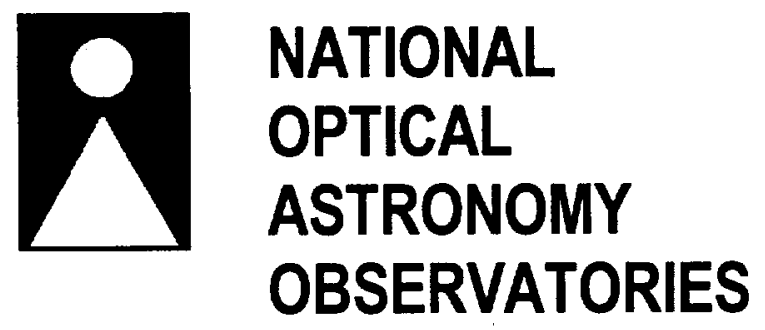

Preprint Series

NOAO Preprint No. 862

COMA MORPHOLOGY DUE TO AN EXTENDED ACTIVE REGION AND IMPLICATIONS FOR THE SPIN STATE OF COMET HALE-BOPP

Nalin H. Samarasinha

(National Optical Astronomy Observatories)

Accepted By: Astrophysical Letters 


\title{
Coma Morphology Due to an Extended Active Region and Implications for the Spin State of Comet Hale-Bopp
}

\author{
Nalin H. Samarasinha \\ National Optical Astronomy Observatories \\ 950 N. Cherry Ave., Tucson, Arizona 85719
}

\begin{abstract}
We show that the circular character of continuum structures observed in the coma of comet Hale-Bopp around the perihelion passage is most likely due to a dust jet from a large extended active region on the surface. Coma morphology due to a wide jet is different from that due to a narrow jet. The latter shows foreshortening effects due to observing geometry, wider jet produces more circular features. This circularization effect provides a self-consistent explanation for the evolution of near-perihelion coma morphology. No changes in the direction of the rotational angular momentum vector are required during this period in contrast to the models of Schleicher et al. This circularization effect also enables us to produce near-circular coma features in the S-E quadrant during 1997 late February and therefore questions the basic premise on which Sekanina bases his morphological arguments for a gravitationally bound satellite nucleus.
\end{abstract}

Subject headings: comets: general — comets: individual (Hale-Bopp C/1995 O1) — methods: numerical

\section{Introduction}

A primary characteristic of the evolving coma morphology of comet Hale-Bopp (C/1995 O1) was the presence of repetitive near-circular dust features around the time of the perihelion passage (e.g., Mueller et al. 1999, and references therein). These features which persisted for nearly a three month period centered around the perihelion passage of 1997 April 1 showed a periodicity of $\approx 11.3$ hrs. This periodicity can be identified with the rotation of the nucleus (Farnham et al. 1998; Licandro et al. 1998; Jorda et al. 1999; Warell et al. 1999).

During the near-perihelion months, images corresponding to emission features $\mathrm{CN}, \mathrm{C}_{2}, \mathrm{C}_{3}, \mathrm{NH}$, and $\mathrm{OH}$ (Larson et al. 1997; Schleicher et al. 1998; Lederer et al. 1999; Mueller et al. 1999) show complete spiral structures around the nucleus. Careful image enhancement by Schleicher

\footnotetext{
${ }^{1}$ The National Optical Astronomy Observatories are operated by the Association of Universities for Research in Astronomy, Inc., under cooperative agreement with the National Science Foundation.
}

et al. (1998) has revealed that the morphology in the continuum images also shows spiral behavior. But the signature therein is only barely evident in the anti-solar direction. These spiral structures were nearly circular Archimedean spirals (i.e., displayed little or no foreshortening effects). They maintained approximately the same shape during the three month period centered around the perihelion when the Earth-comet-sun (E-C-S) geometry changed significantly.

Attempts have been made to determine whether the nucleus is in a principal axis state or not as well as to fix the direction of the rotational angular momentum vector, M (Sekanina 1998a; Jorda et al. 1999; Licandro et al. 1999; Samarasinha et al. 1999; Vasundhara \& Chakraborty 1999). Simulations aimed at replicating near-perihelion coma morphology have encountered many difficulties (Sekanina 1998b; Vasundhara \& Chakraborty 1999) and Sekanina has argued that two gravitationally bound active nuclei might explain the evolution of near-perihelion coma morphology.

Schleicher et al. $(1998,1999)$, in their attempts to mimic the evolution of near-perihelion coma 
morphology, deduced a greater than $90^{\circ}$ precession of the axis of the "cone" generated by the relevant active region on the rotating nucleus. This precession has occurred over a three month period. Furthermore, this "cone axis", which can be identified with the vector $M$ in the case of a principal axis rotator, approximately followed the direction of Earth which also moved nearly $90^{\circ}$ over the said period, an unlikely or extremely fortuitous situation.

In this Letter we present new coma simulations that include a hitherto unexplored projection effect which gives a self-consistent explanation for the evolutionary behavior of near-perihelion coma morphology without involving exceptional physical or dynamical situations.

We first show (section 2) that the coma morphology due to observationally compatible complex spin states are qualitatively the same as that due to a principal axis rotator. In section 3 we determine the probable rate of forced precession of $\mathbf{M}$ due to outgassing from the nucleus and show that it is unlikely to have moved more than few degrees during the 1997 March-May period. In section 4 we calculate the total area of the active regions on the nucleus and demonstrate that the dominant active region must have considerable angular extension $\left(\approx 40^{\circ}\right)$. Numerical simulations that emulate the coma morphology due to such an active region are then presented that show that a precession of $M$ is not required to explain what is observed. Section 5 discusses certain additional issues which are of relevance to coma morphology.

\section{Can a Non-Principal Axis Spin State Explain the Evolution of Coma Mor- phology?}

During the 1997 March-May period, the nearcircular spiral pattern of the coma morphology was nearly unchanged whereas the Earth direction (and the solar direction) moved by nearly $90^{\circ}$. To explain this Schleicher et al. $(1998,1999)$ proposed a continual precession of the vector $M$ over this three month period.

An immediate question that arises is whether a non-principal axis rotation state for the nucleus of comet Hale-Bopp can provide a more simple explanation for this behavior. Samarasinha et al. (1999) identified two categories of non- principal axis spin states which can reproduce regular repetitive coma structures. They are (a) a spin state with a small precession angle of the order of few degrees, and (b) a spin state in which one component period is several orders of magnitude larger than the other period. For this latter case, the shorter period corresponds to the precession of the angular velocity vector around the angular momentum vector, $\mathbf{M}$ (Ames \& Murnaghan 1929; Samarasinha et al. 1999). For both of these complex spin scenarios, the angular velocity vector, the angular momentum vector, and the "cone axis" must be aligned within few degrees of each other. As in the case for the principal axis states, in all observationally compatible non-principal axis states, the "cone axis" can for all practical purposes be approximately identified with the vector $\mathbf{M}$. Therefore, if a precession of the vector $\mathbf{M}$ is invoked for a principal axis rotator to explain the evolutionary behavior of near-perihelion coma morphology, the same holds true for the observationally compatible complex spin states.

This does not mean there are no morphological differences between coma structures generated by a principal axis rotation and an observationally compatible complex rotation. Only that the "cone axis" will in both cases be nearly aligned with $\mathbf{M}$ all the time. For example, the rotation associated with the longer period of a complex spin state with a large ratio between component periods (the second complex spin scenario described earlier), would result in variations in the opening angle of the "cone" with time. This may manifest itself as a time dependent (related to the longer component period) variations in the projected outflow velocity of the coma structures. Thus, irrespective of whether a simple or a complex rotator is involved in the type of modeling done by Schleicher et al. $(1998,1999)$, the result will imply a large precession of $\mathbf{M}$.

\section{Dynamical Constraints on the Evolu- tion of Angular Momentum}

The e-folding time scale, $\tau_{\mathbf{M}}$, for changes in $\mathbf{M}$ due to reaction torques caused by outgassing is given by (Samarasinha et al. 1986; Jewitt 1992)

$$
\tau_{\mathrm{M}}=k \frac{\omega \rho r^{5}}{d V \dot{m}}
$$


where $P(=2 \pi / \omega)$ is the rotation period, $\rho$ is the bulk density of the nucleus, $r$ is the nuclear radius, $d$ is the effective moment arm for the net torque, $V$ is the gas outflow velocity at the nucleus, $m$ is the gas production rate, and $k$ is a constant with a value of about 2 .

If $d$ is taken to be $r$, we obtain a strict lower limit to $\tau_{\mathrm{M}}$. By adopting $P=11.3 \mathrm{hr}, \rho=0.4 \mathrm{~g}$ $\mathrm{cm}^{-3}, r=30 \mathrm{~km}$ (Weaver \& Lamy 1999; Y. Fernandez et al., in preparation), $V=1 \mathrm{~km}$ $\mathrm{s}^{-1}$, and $\dot{m}=3 \times 10^{8} \mathrm{~g} \mathrm{~s}^{-1}$ (corresponding to $Q_{\mathrm{H}_{2} \mathrm{O}}=10^{31}$ molecules s${ }^{-1}$ (Biver et al. 1999)), we arrive at $\tau_{M}>10$ yrs. A more realistic value for $d$ may be smaller by about 1 order of magnitude (Jewitt 1999) and therefore $\tau_{\mathbf{M}}$ is more likely to be of the order of 100 yrs.

In addition, prior studies on spin state evolution show that large changes in $\mathbf{M}$, due to outgassing torques, are accompanied by correspondingly large changes in the rotation period $P$ (Samarasinha \& Belton 1995). However, in the case of comet HaleBopp, no such change in the $11.3 \mathrm{hr}$ periodicity during the 1997 March-May period was detected (Schleicher et al. 1998). Therefore, it is most unlikely that the direction of $\mathrm{M}$ would have changed by more than few degrees during the approximate three month period around the perihelion.

\section{Coma Morphology Due to an Extended Active Region}

\subsection{Size of Active Regions on Hale-Bopp}

We now examine whether the existing coma simulations, all based on point source or narrow jet models, are reasonable.

First, we estimate the size of a single active region which could be responsible for the dominant coma feature. We adopt a perihelion water production rate of $10^{31}$ molecules $\mathrm{s}^{-1}$ (Biver et al. 1999) corresponding to a perihelion distance of $0.91 \mathrm{AU}$. Based on simple thermal models (Cowan \& A'Hearn 1979; Julian et al. 1999), this corresponds to an area of approximately $2 \times 10^{3} \mathrm{~km}^{2}$ for the active region. i.e., an active region (or group of regions) which occupies $10 \%$ or more of the total surface area if the upper limit to the nuclear radius is taken to be $40 \mathrm{~km}$ (Weaver \& Lamy 1999). If only a fraction, say $40 \%$, of the dust production occurs in the single dominant active region, then it would have an angular extension of the order of $40^{\circ}$. The exact angular extensions would, of course, depend on the shape of the active region and the actual nucleus radius itself.

This contrasts with the existing simulations of coma features in Hale-Bopp, including those by the author himself, which have usually been carried out assuming either a point source model for the active region or a narrow jet. The physical situation questions this approach and we reexamine the coma morphology using a large extended active region as the basis of the modeling.

\subsection{Simulations of Coma Morphology}

Figure 1 shows the dependence of coma morphology for point source dust jets with different gaussian angular cross sectional widths. For all these cases, the observer is inside the "cone" swept out by the jet axes due to the rotation of the nucleus, the scenario corresponding to the near perihelion time. Since we are simulating coma structures with an age of the order of only several rotation periods, and we are interested in identifying the behavior of coma morphology as a function of the jet width, radiation pressure effects are not considered. It is found that the spiral structure becomes more circularized as the jet width increases. i.e., as the jet width increases, the foreshortening effects are reduced and the perceived length of the "minor axis" approaches that of the "major axis".

The following qualitative explanation will further clarify this non-intuitive jet-width dependent projection effect.

For a narrow jet, foreshortening occurs in the "short axis" direction defined by the intersection of the sky plane and the plane which contains the line-of-sight (LOS) and M. Consider dust grains ejected during a short interval when the jet is in the plane containing the LOS and M. The resultant image would show a small dust patch in the direction of the "short axis". The apparent outHow velocity for that dust patch would be $V_{d} \sin \theta$, where $V_{d}$ is the radial dust outflow velocity and $\theta$ is the angle between the LOS and the direction at which dust was ejected. Now, consider a wide jet where all these ejected dust grains are spread over a shell of radius $R$ defined by the dust outflow velocity $V_{d}$. If the angular distribution of dust is sufficiently wide, the peak brightness in an image in the direction of the "short axis" would occur 
further out than in the narrow jet because of the extension of the shell along the LOS. This description crudely illustrates how the foreshortening effects are reduced when you have a wide jet.

An alternative way of demonstrating this effect is shown in figure 2 where we show the coma morphology due to dust from an extended active region. To simulate this morphology, we co-added five point source model jets which are placed equi distant within the extended active region. Images in figure 2 mimic the dust outflow from an extended active region on Hale-Bopp at two widely separated times and show how a nearly circular coma morphology is maintained. Despite large changes in the E-C-S geometry, this morphology maintains its circular character over time scales of months around the perihelion time consistent with observations.

This circularization of coma features by a wide jet provide a natural self-consistent explanation for the structures observed in Hale-Bopp over approximately three months around the perihelion without requiring any precession of $\mathbf{M}$.

In addition, as we show in figure 3 , outgassing from a wide extended area, unlike a narrow jet, generates the near-circular coma structures in the S-E quadrant during 1997 February 23 for the $M$ direction assumed by Sekanina (1998b). This result questions the basic premise upon which he has argued for two active nuclei for comet Hale-Bopp.

\section{Discussion}

\subsection{Radial Outflow Velocity of Dust}

A comparison of coma features due to jets of different widths (figure 1) indicate that the circularization occurs as a reduction of foreshortening effects in the direction normal to the maximum width of the feature. For wide jets, the maximum width of the features corresponds to the radial outflow velocity of dust. Therefore, separation between repetitive structures in the direction of maximum width is a direct measure of the radial dust outflow velocity.

\subsection{Other Factors Affecting Coma Mor- phology}

The simulations presented in this Letter assume that the dust jets maintain the same angular cross sectional widths irrespective of cometocentric distance. Hydrodynamical simulations, especially that close to the nucleus (Kitamura 1990; Crifo \& Rodionov 1999, and references therein) indicate the possibility of substructures due to shock formation if there are several spatially separated source regions. In addition, if the whole active region is not outgassing uniformly but has localized high activity at certain parts, then that could also be responsible for some of the substructure. It is not clear how these effects will be propagated out to the observable coma and relevant calculations are yet to be carried out, a task beyond the scope of this Letter. Here, we assume that they would only cause secondary effects and indeed some of the fainter substructures seen in the coma morphology of comet Hale-Bopp may be related to such effects. A detailed examination of hydrodynamical effects close to the nucleus and their possible influence on the observable coma morphology will be discussed elsewhere (M. Belton et al., in preparation).

An increase in the angular cross sectional width of dust jets in the circumnuclear region as a function of increasing cometocentric distance is demonstrated by Combi et al. (1999) based on their hydrodynamical simulations. Therefore, the angular cross sectional width of a dust jet at observable distances may in fact be larger than its original angular width at the nucleus, again justifying the choice of a wide jet.

\subsection{Coma Morphology Due to Multiple Active Regions}

In addition to the spiral coma structure due to a single dominant active region, there are at least one (Schleicher et al. 1998) or perhaps two (Licandro et al. 1998; Farnham et al. 1999) jet features which intersect with the primary feature at shallow angles for each repetitive cycle. As narrower jets would show different curvatures than the curvature of the wider dominant jet, these secondary jets are likely to be originating from smaller active regions on the surface. These need to be taken into account during future detailed coma modeling. 


\section{Summary}

Coma morphology due to a wide jet is shown to be substantially different from that due to a narrow jet. The morphology due to a narrow jet shows strong foreshortening effects due to observing geometry, while a wider jet produces more circular features.

This jet-width dependent projection effect has the following immediate implications.

(1) The nearly unchanged repetitive circular spiral structures observed over approximately three months in Hale-Bopp can be explained most simply by a wide jet originating from a large extended active region on the nucleus. Little, or no, change in the direction of $\mathbf{M}$ during this time interval is required. This scenario is consistent with dynamical calculations.

(2) Contrary to the claim of Sekanina (1998b), simulations indicate the existence of near-circular coma features in the S-E quadrant during 1997 late February. This seriously questions the basic premise upon which he bases his morphological arguments for two active nuclei.

(3) The separation between the repetitive cycles of the coma structure in the direction of maximum width is a direct measure of the radial dust outflow velocity.

I thank Dr. M. Belton for many helpful suggestions, Dr. B. Mueller for going through an early draft, and Drs. D. Schleicher and Z. Sekanina for discussions on their work. I thank the referee, Dr. T. Farnham for his valuable comments. This work was supported by NASA Planetary Astronomy Program.

\section{REFERENCES}

Ames, J.S. \& Murnaghan, F.D. 1929, Theoretical Mechanics (Boston, Ginn \& Co.)

Biver, N., et al. 1999, Earth Moon Planets, in press.

Cowan, J.J. \& A'Hearn, M.F. 1979, Moon Planets, $21,155$.

Combi, M.R., Kabin, K., DeZeeuw, D.L., \& Gombosi, T. 1999, Earth Moon Planets, in press.

Crifo, J.F., \& Rodionov, A.V. 1999, Planet. Space Sci., 47, 797.

Farnham, T.L., Schleicher, D.G., \& Cheung, C.C. 1998, BAAS, 30, 1072.

Farnham, T.L., Schleicher, D.G., Williams, W.R, \& Smith, B.R. 1999, BAAS, 31, 1120.

Jewitt, D. 1992, in Proceedings of the $30^{\text {th }}$ Leige International Astrophysical Colloquium, ed. A. Brahic, J.-C. Gerard, \& J. Surdej (Univ. of Leige press), 85 .

Jewitt, D. 1999, Earth Moon Planets, in press.

Jorda, L., Rembor, K., Lecacheux, J., Colom, P., Colas, F., Frappa, F. \& Lara, L.M. 1999, Earth Moon Planets, 77, 167.

Julian, W.H., Samarasinha, N.H., \& Belton, M.J.S. 1999, Icarus, in press.

Kitamura, Y. 1990, Icarus, 86, 455.

Larson, S.M., Hergenrother, C.W., \& Brandt, J.C. 1997, BAAS, 29, 1036.

Lederer, S.M., Campins, H., Osip, D.J., \& Schleicher, D.G. 1999, Earth Moon Planets, in press.

Licandro, J., et al. 1998, ApJ, 501, L221.

Licandro, J., et al. 1999, Earth Moon Planets, 77, 199.

Mueller, B.E.A., Samarasinha, N.H., \& Belton, M.J.S. 1999, Earth Moon Planets, 77, 181.

Samarasinha, N.H., A'Hearn, M.F., Hoban, S., \& Klinglesmith, D. 1986, in $20^{\text {th }}$ ESLAB Symp. on the Explo. of Halley's Comet (ESA SP-250 Vol. 1), 487. 
Samarasinha, N.H., \& Belton, M.J.S. 1995, Icarus, 116,340 .

Samarasinha, N. H., Mueller, B.E.A., \& Belton, M.J.S. 1999, Earth Moon Planets, 77, 189.

Sekanina, Z. 1998a, ApJ, 494, L121.

Sekanina, Z. 1998b, ApJ, 509, L133.

Schleicher, D. G., Farnham, T.L., Smith, B.R., Blount, E.A., Nielsen, E., \& Lederer, S.M. 1998, BAAS, 30, 1063.

Schleicher, D. G., Farnham, T.L., Smith, B.R., \& Cheung, C.C. 1999, in Abstracts of Asteroids Comets Meteors 1999 Meeting, 114.

Vasundhara, R., \& Chakraborty, P. 1999, Icarus, $140,221$.

Warell, J., Lagerkvist, C.-I., \& Lagerros, J.S.V. 1999, A\&A Supp., 136, 245.

Weaver, H.A., \& Lamy, P.L. 1999, Earth Moon Planets, in press.

This 2-column preprint was prepared with the AAS IATEX macros v5.0.
Fig. 1.- Simulated coma morphology as seen from Earth for the Earth-comet-sun geometry of 1997 March 13 for dust jets having standard deviation, $\sigma$, of $1^{\circ}, 5^{\circ}, 15^{\circ}$, and $30^{\circ}$ (clockwise from top left) for the gaussian cross sectional width. For all four cases, the other parameters were kept unchanged. Earth is inside the "cone" swept out by the axes of the jets. For each panel, the nucleus is at the center. North is up and east is to the left. A single dust outflow velocity is assumed. The dust production rate is assumed to be proportional to $(0.1+\cos \alpha)$ during day time and 0.1 during the night, where $\alpha$ is the solar zenith angle. This night time emission, which helps the eye to trace the spiral structure, is primarily confined to an approximately $90^{\circ}$ sector in the westerly direction.

Fig. 2.- Simulated coma morphology as seen from Earth for E-C-S geometries of 1997 March 13 (left) and 1997 May 6 (right) for otherwise identical parameters. Point source dust jets of $\sigma$ of $15^{\circ}$ originating from a five point equi-distant grid located within an extended active region are coadded in each image. The five points are located at the center of the active region (same physical location as that in figure 1), at longitude displacements of $\pm 20^{\circ}$, and at latitude displacements of $\pm 20^{\circ}$. For both times, Earth is inside the "cone" swept out by the axis of the central jet. Same dust production rate as in figure 1 is used. Spatial coverage in May image is larger since the geocentric distance is larger.

Fig. 3.- Simulated coma morphology as seen from Earth due to a jet of $\sigma$ of $1^{\circ}$ (left) and that due to an extended active region (right) for 1997 February 23 for the same M direction of Sekanina (1998b). We used a five point grid similar to that described in the caption for figure 2 to mimic the dust outflow from an extended active region. The origin of the point source jet on the left as well as the center point of the extended active region are located at latitude $+10^{\circ}$. For each panel, the nucleus is at the center. North is up and east is to the left. Zero dust production is assumed during the night time. Notice that the image on the left corresponding to a narrow jet is much like what Sekanina (1998b, figure 3) obtained, while an extended active region produces near-circular features in the S-E quadrant. 

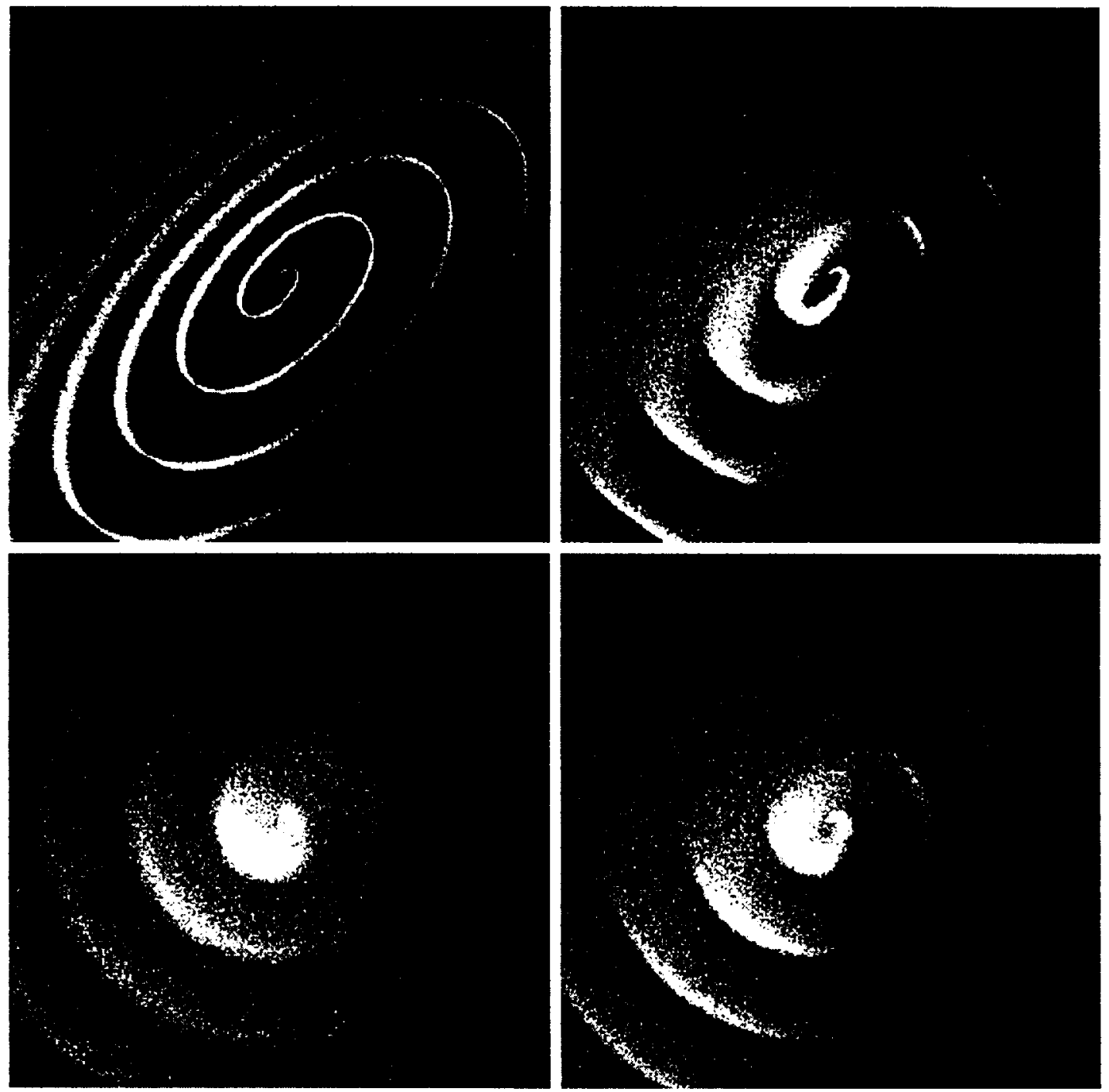

Fig 1 


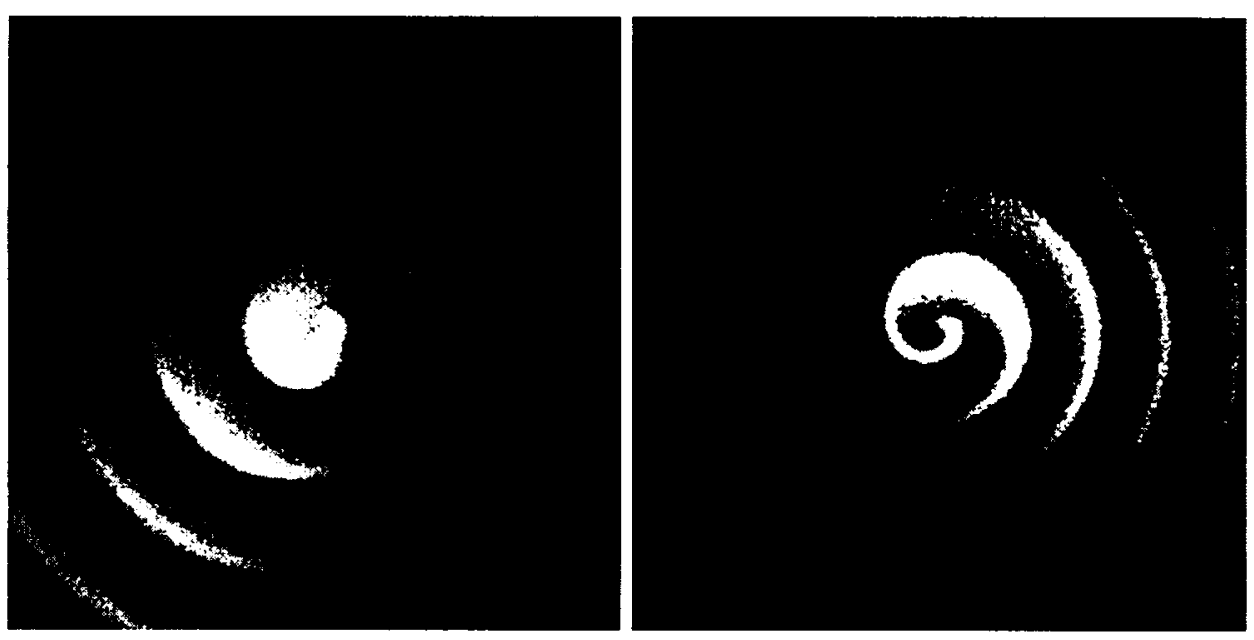

Fig 2 


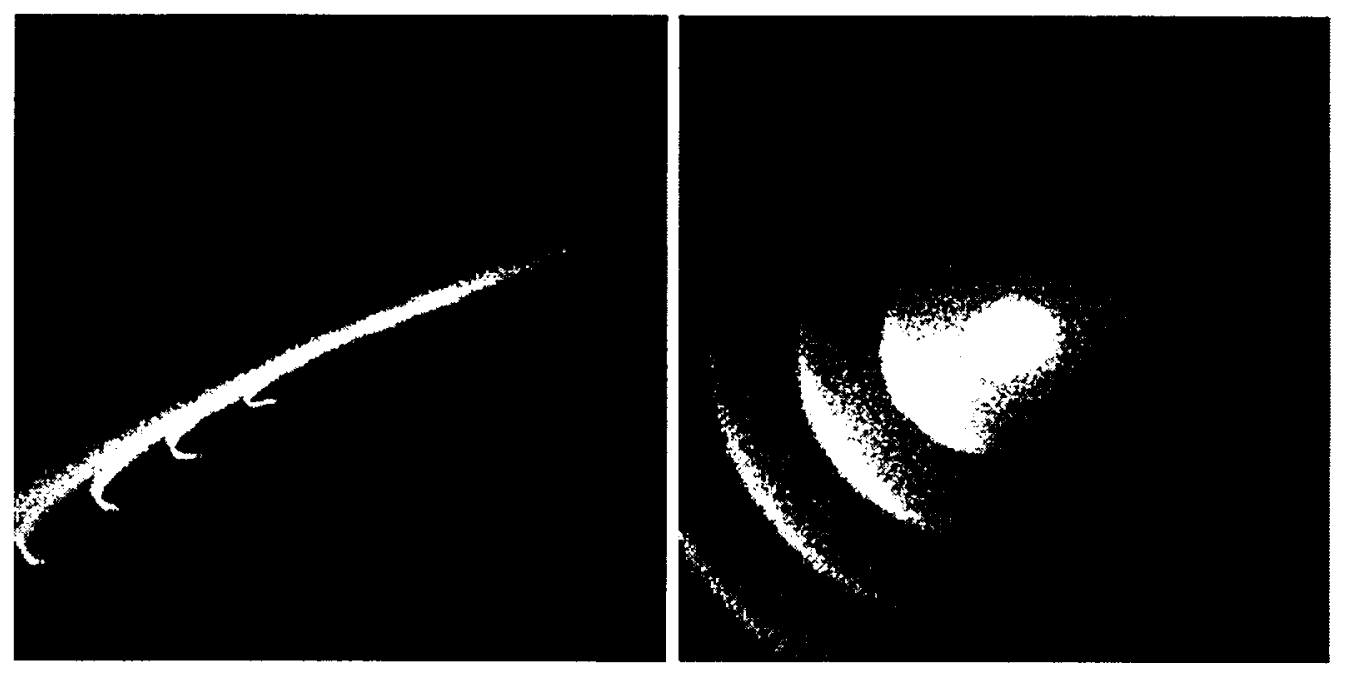

Fig 3 